\title{
An Iterative Technique for Solving a Class of Nonlinear Quadratic Optimal Control Problems Using Chebyshev Polynomials
}

\author{
Husse in Jaddu, Amjad Majdalawi \\ Electronics Engineering Department, Faculty of Engineering, Al Quds University, Jerusalem, Palestine \\ Email: jaddu@eng.alquds.edu, amjadmajdalawi@hotmail.com
}

\begin{abstract}
In this paper, a method for solving a class of nonlinear optimal control problems is presented. The method is based on replacing the dynamic nonlinear optimal control problem by a sequence of quadratic programming problems. To this end, the iterative technique developed by Banks is used to replace the original nonlinear dynamic system by a sequence of linear time-varying dynamic systems, then each of the new problems is converted to quadratic programming problem by parameterizing the state variables by a finite length Chebyshev series with unknown parameters. To show the effectiveness of the proposed method, simulation results of a nonlinear optimal control problem are presented.
\end{abstract}

Index Terms - Nonlinear quadratic optimal control problem, Banks Iterative Technique, Chebyshev polynomials, State parameterization

\section{INTRODUCTION}

Many direct methods have been developed to solve the nonlinear optimal control problems. All direct methods are based on converting the dynamical optimal control problem into static optimization problem, These methods are either based on discretization [1,2] or on parameterization. The parameterization can be implemented by parameterizing the state variables [3-5], control variables [6,7] or both the state and control variables $[8,9]$.

Jaddu [3-5] proposed a method to solve the nonlinear optimal control problem using the quasilineraization and the Chebyshev polynomials. In [10-14] Banks and his coauthers developed a method to deal with the nonlinear systems by means of iterative approach.

In this paper, we propose a new method based on the Banks iterative technique to replace the nonlinear optimal control problem by sequence of linear time-varying quadratic optimal control problems. Then each of these problems is converted into quadratic programming problem. To this end, the Chebyshev polynomials are used to approximate the state variables. The proposed method in this paper is classified as a direct method. Approximating the state variables has the following advantages [3]: (1) There is no need to integrate the system state equations as in control parameterization. (2) The number of unknown parameters is smaller compared with control-state parameterization. (3) The state constraints can be handled directly; an example of this in this work is the approximation of the initial condition vector.
This paper is organized as follows: In section 2, the problem treated in the paper is stated. Section 3, shows how to apply the Banks iterative method on the problem of section 1 . Section 4 describes the state parameterization and the approximation by Chebyshev polynomials. In addition, this section, shows the transformation of the dynamic optimal control problem into quadratic programming problems. Section 5 shows the simulation results of the Van der Pol oscillator optimal control problem and compares the results obtained using the proposed technique with other techniques. In section 6 some conclusion remarks are presented.

\section{PROBlem ST ATEMENT}

The optimal control problem treated in this paper can be stated as follows: Find an optimal controller $u^{*}(t)$ that minimizes the following performance index

$$
J=\int_{0}^{t_{f}}\left(x^{T} Q x+u^{T} R u\right) d t
$$

subject to the constraints of the system state equations and initial conditions given by,

$$
\dot{x}=A(x) x+B(x) u \quad x(0)=x_{0}
$$

where $\mathrm{Q}$ is a positive semidefinite matrix, $R$ is a positive definite matrix, $x \in R^{n}$ is the state vector, $\mathrm{u}$ $\in R^{m}$ is the control vector, $x_{0} \in R^{n}$ is the initial condition vector.

This problem will be solved by converting the nonlinear quadratic optimal control problem into a sequence of quadratic programming problems, which are easier to solve. The solution is based on using the iteration technique, which will replace the nonlinear quadratic optimal control problem into equivalent sequence of linear time-varying quadratic optimal control problems. Then, each of these problems will be solved by converting it into a quadratic programming problem by using state parameterization via Chebyshev polynomials.

\section{BANKS ITERATIVE TECHNIQUE}

In this section, the iterative technique developed by Banks [10-14] is applied on the problem (1)-(2) to convert this problem into a sequence of linear time-varying quadratic optimal control problems as follows: 
For $\mathrm{i}=0$

Minimize

$$
J^{[0]}=\int_{0}^{t f}\left(x^{[0]^{T}} Q x^{[0]}+u^{[0]^{T}} R u^{[0]}\right) d t
$$

subject to the following state equations and initial conditions

$$
\dot{x}^{[0]}=A\left(x_{0}\right) x^{[0]}+B\left(x_{0}\right) u^{[0]} \quad x^{[0]}(0)=x_{0}
$$

and for $i \geq 1$

\section{Minimize}

$$
J^{[i]}=\int_{0}^{t} f\left(x^{[i]^{T}} Q x^{[i]}+u^{[i]^{T}} R u^{[i]}\right) d t
$$

subject to the following state equations and initial conditions

$$
\begin{aligned}
& \dot{x}^{[i]}=A\left(x^{[i-1]}(t)\right) x^{[i]}+B\left(x^{[i-1]}(t)\right) u^{[i]} \\
& x^{[i]}(0)=x_{0}
\end{aligned}
$$

Each of the linear time-varying quadratic optimal control problem (3)-(6) is converted into a quadratic programming problems by applying the state parameterization technique using Chebyshev polynomials . Therefore, the time interval $t \in\left[0, t_{f}\right]$ should be changed to $\tau \in[-1,1]$ because Chebyshev polynomials are orthogonal when defined of the interval $\tau \in[-1,1]$. This will transform the problems (3)-(6) into

For $\mathrm{i}=0$

Minimize

$$
J^{[0]}=\frac{t_{f}}{2} \int_{-1}^{1}\left(x^{[0]^{T}} Q x^{[0]}+u^{[0]^{T}} R u^{[0]}\right) d \tau
$$

subject to:

$$
\begin{aligned}
\frac{d x^{[0]}}{d \tau} & =\frac{t_{f}}{2}\left[A\left(x_{0}\right) x^{[0]}+B\left(x_{0}\right) u^{[0]}\right] \\
x^{[0]}(-1) & =x_{0}
\end{aligned}
$$

and for $i \geq 1$

Minimize

$$
J^{[i]}=\frac{t_{f}}{2} \int_{-1}^{1}\left(x^{[i]^{T}} Q x^{[i]}+u^{[i]^{T}} R u^{[i]}\right) d \tau
$$

subject to:

$$
\begin{aligned}
& {\frac{d x^{[i]}}{d \tau}}^{[i]}=\frac{t_{f}}{2}\left[A\left(x^{[i-1]}(\tau)\right) x^{[i]}+B\left(x^{[i-1]}(\tau)\right) u^{[i]}\right], \\
& x^{[i]}(-1)=x_{0}
\end{aligned}
$$

\section{CHEBYShEV APPROXIMATION}

To convert each of the optimal control problems (7)(10) into a quadratic programming problem, a set of the state variables are approximated by a finite length Chebyshev series with unknown parameters. Then, the remaining states and control variables are determined as a function of the unknown parameters of the approximated state variables from the state equations (8) and (10). In [15] a detailed description of how to apply the state parameterization using Chebyshev polynomials is presented.

Applying the method described in [15], the state variables can be approximated by using the Chebyshev polynomials of the first type as follows

$$
x_{j}=\frac{a_{0}^{(j)}}{2}+\sum_{i=1}^{N} a_{i}^{(j)} T_{i}(\tau) \quad j=1,2, \ldots, n
$$

where $N$ is the length of the approximated series which depends on the required accuracy; large values of $N$ y ield good accuracy at the expense of computational time and vice versa, $a_{i}$ are the unknown parameters and $T_{i}$ is a first type Chebyshev polynomial of order $i$. The control variables are obtained from the system state equations as a function of the state variables unknown parameters. These control variables can be rewritten in terms of a finite length series of Chebyshev polynomials with unknown parameters $b_{i}$ as follows

$$
u_{l}=\frac{b_{0}^{(l)}}{2}+\sum_{i=1}^{N} b_{i}^{(l)} T_{i}(\tau) \quad l=1,2, \ldots, m
$$

where the unknown parameters $b_{i}^{(l)}$ are function of the state variables unknown parameters $a_{i}^{(j)}$.

In state parameterization, it is necessary to obtain the derivative of the state variables. This can be done using Chebyshev polynomials differentiation properties as follows [16]

$$
\dot{x}_{j}(\tau)=\frac{a_{0}}{2}+\sum_{i=1}^{N-1} a_{\imath} T_{i}(\tau) \quad j=1,2, \ldots, n
$$

where

$$
\left\{\begin{array}{l}
a_{N-1}=2 N a_{N} \\
a_{N-2}=2(N-1) a_{N-1} \\
a_{r-1}=a_{r+1}+2 r a_{r}, r=1,2, \ldots, N-2
\end{array}\right.
$$

Equation (10) shows that the two matrices $A\left(x^{[i-1]}(\tau)\right)$ and $B\left(x^{[i-1]}(\tau)\right)$ are a function of $\tau$, therefore it is necessary to express every element in both matrices in terms of a Chebyshev series of known parameters. To this end, let $A_{j l}(\tau)=g\left(x^{[i-1]}(\tau), \tau\right)$ be the $(j, l)$ element of the matrix $A\left(x^{[i-1]}(\tau)\right)$ where $x^{[i-1]}(\tau)$ is the nominal trajectory of the previous iteration. Then the term $A_{j l}(\tau)$ can be expressed in terms of a Chebyshev series of known parameters of the form [16]

$$
A_{j l}(\tau)=\frac{W_{0}}{2}+\sum_{i=1}^{M} W_{i} T_{i}(\tau)
$$

where

$$
\begin{aligned}
& W_{j}=\frac{2}{K} \sum_{i=1}^{K} g\left(\cos \left(\theta_{i}\right)\right) \cos \left(j \theta_{i}\right) \\
& j=0,1, \ldots, N
\end{aligned}
$$


and $\theta_{i}=\frac{2 i-1}{2 K} \pi, i=1,2, \ldots, K$ and $K>N$ (in this work we arbitrary set $K=N+5)$. The same approximation can be done for the matrix $B\left(x^{[i-1]}(\tau)\right)$.

Also equation (10) shows that $A\left(x^{[i-1]}(\tau)\right)$, $B\left(x^{[i-1]}(\tau)\right)$ are time-varying matrices expressed as a function of Chebyshev polynomials of the previous iteration. The matrix $A\left(x^{[i-1]}(\tau)\right)$ is multiplied by $x^{[i]}$, which is also expressed in terms of Chebyshev series with unknown parameters, and the matrix $B\left(x^{[i-1]}(\tau)\right)$ is multiplied by $u^{[i]}$, which is also expressed as a function of Chebyshev series with unknown parameters. Therefore, it is necessary to have a multiplication algorithm to multiply Chebyshev series. This algorithm is given as follows [3]:

Given two Chebyshev series

$$
\begin{gathered}
X=\sum_{i=0}^{n} x_{i} T_{i} \\
\mathrm{Y}=\sum_{\mathrm{j}=0}^{\mathrm{m}} \mathrm{y}_{\mathrm{j}} \mathrm{T}_{\mathrm{j}}
\end{gathered}
$$

Then the multiplication of these two Chebyshev series is a Chebyshev series of length $n+m$ given by

$$
\sum_{k=0}^{n+m} Z_{k} T_{k}
$$

where

$$
z_{k}=\frac{1}{2} \sum_{i=0}^{n} x_{i} y_{k-i}+x_{i} y_{i-k}+x_{i} y_{i+k}
$$

The next step is to approximate the initial condition vector $x(-1)=x_{0}$ and since we are using state parameterization, this task becomes very easy. By substituting $\tau=-1$ into (11) and using the following Chebyshev initial value property [16],

$$
T_{n}(-1)=(-1)^{n}
$$

the initial condition vector is approximated as follows

$$
\frac{a_{0}^{(j)}}{2}-a_{1}^{(j)}+a_{2}^{(j)}-\cdots+(-1)^{N} a_{N}^{(j)}-x_{j}(-1)=0
$$

The last step is to approximate the performance index $\mathbf{J}$. To do this, (11) and (12) are rewritten in matrix form

$$
\begin{aligned}
& {\left[\begin{array}{c}
x_{1} \\
x_{2} \\
\vdots \\
x_{n}
\end{array}\right]=\left[\begin{array}{cccc}
0.5 a_{0}^{(1)} & a_{1}^{(1)} & \ldots & a_{N}^{(1)} \\
0.5 a_{0}^{(2)} & a_{1}^{(2)} & \ldots & a_{N}^{(2)} \\
\vdots & \vdots & \vdots & \vdots \\
0.5 a_{0}^{(n)} & a_{1}^{(n)} & \ldots & a_{N}^{(n)}
\end{array}\right]\left[\begin{array}{c}
T_{0} \\
T_{1} \\
\vdots \\
T_{N}
\end{array}\right]} \\
& {\left[\begin{array}{c}
u_{1} \\
u_{2} \\
\vdots \\
u_{m}
\end{array}\right]=\left[\begin{array}{cccc}
0.5 b_{0}^{(1)} & b_{1}^{(1)} & \ldots & b_{N}^{(1)} \\
0.5 b_{0}^{(2)} & b_{1}^{(2)} & \ldots & b_{N}^{(2)} \\
\vdots & \vdots & \vdots & \vdots \\
0.5 b_{0}^{(m)} & b_{1}^{(m)} & \ldots & b_{N}^{(m)}
\end{array}\right]\left[\begin{array}{c}
T_{0} \\
T_{1} \\
\vdots \\
T_{N}
\end{array}\right]}
\end{aligned}
$$

or in compact form

$$
x^{[i]}=S_{1} T \quad u^{[i]}=S_{2} T
$$

Substituting (25) into equation (7) (for the first iteration) or equation (9) (for the next iterations), we get

$$
\hat{\mathrm{J}}^{[\mathrm{i}]}=\frac{\mathrm{t}_{\mathrm{f}}}{2} \int_{-1}^{1}\left(\mathrm{~T}^{\mathrm{T}} \mathrm{S}_{1}{ }^{\mathrm{T}} \mathrm{Q} \mathrm{S}_{1} \mathrm{~T}+\mathrm{T}^{\mathrm{T}} \mathrm{S}_{2}{ }^{\mathrm{T}} \mathrm{RS}_{2} \mathrm{~T}\right) \mathrm{d} \tau
$$

where $\hat{J}^{[i]}$ is the approximated value of $J$ at iteration $i$. By letting $M=S_{1}{ }^{T} Q S_{1}$ and $P=S_{2}{ }^{T} R S_{2}$ and noting that both matrices $M$ and $P$ are symmetrical, Jaddu [3,15] derived an explicit formula for the approximated performance index $\hat{f}^{[i]}$ given by,

$$
\begin{gathered}
\hat{J}^{[i]}=t_{f} \sum_{i=1}^{N+1-k} \frac{1}{2}\left(p_{i, i+k}+m_{i, i+k}^{\prime}\right)\left(\frac{-2}{(2 i-2+k)^{2}}+\right. \\
\left.\frac{-2}{k^{2}-1}\right)
\end{gathered}
$$

where

$$
\begin{gathered}
\dot{p}_{i, i+k}= \begin{cases}p_{i, i+k} & k \neq 0 \\
\frac{p_{i i}}{2} & k=0\end{cases} \\
\dot{m}_{i, i+k}= \begin{cases}m_{i, i+k} & k \neq 0 \\
\frac{m_{i i}}{2} & k=0\end{cases}
\end{gathered}
$$

where $k=0,2,4, \ldots, N(N$ even $)$ or $N-1(N$ odd $)$ and $p_{i, j}, m_{i j j}$ are the elements of the symmetrical matrices $P$ and $M$ respectively.

The performance index in (27) can be rewritten as,

$$
\hat{\jmath}=\frac{1}{2} a^{T} H a
$$

where

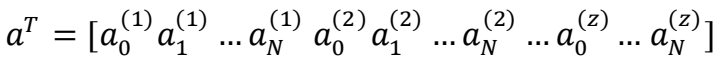

is the unknown parameter vector and $H$ is a ppositive definite [3] Hessian matrix given by

$$
H=\frac{\partial^{2} \hat{f}}{\partial a_{i}^{(k)} \partial a_{j}^{(k)}}
$$

where $i_{j} j=0,1, \ldots, N$ and $k=1,2, \ldots, z$. z is the number of directly approximated state variables.

The original nonlinear quadratic optimal control problem can now be restated as follows:

$$
\min _{a} \quad \frac{1}{2} a^{T} H a
$$

subject to

$$
F_{1} a=b_{1}
$$

where the equality constraints are due to initial conditions and in some cases unsatisfied state equations.

The standard quadratic programming problem (32)-(33) can be solved using any available software package.

To solve the original nonlinear problem (1) -(2), we need to solve time-varying linear quadratic optimal control problem (7)-(10) iteratively until

$$
\left|\hat{J}^{[i+1]}-\hat{J}^{[i]}\right|<\epsilon
$$

is satisfied. In this work we consider $\epsilon=10^{-5}$. 


\section{COMPUtATION RESUlts}

\section{- Van der Pol Oscillator}

Find an optimal controller $u^{*}(t)$ that minimizes the following performance index

$$
J=\frac{1}{2} \int_{0}^{5}\left(x_{1}^{2}+x_{2}^{2}+u^{2}\right) d t
$$

subject to

$$
\begin{aligned}
& \dot{x}_{1}=x_{2} \quad, x_{1}(0)=1 \\
& \dot{x}_{2}=-x_{1}+\left(1-x_{1}^{2}\right) x_{2}+u \quad, x_{2}(0)=0
\end{aligned}
$$

Using the technique in sections 3 and 4 , the problem can be reformulated as:

for $i=0$

Minimize

$$
J^{[0]}=\frac{1}{2} \int_{0}^{5}\left(\left(x_{1}^{[0]}\right)^{2}+\left(x_{2}^{[0]}\right)^{2}+\left(u^{[0]}\right)^{2}\right) d t
$$

subject to

$$
\begin{gathered}
\dot{x}_{1}{ }^{[0]}=x_{2}{ }^{[0]} \quad, x_{1}{ }^{[0]}(0)=1 \\
\dot{x}_{2}{ }^{[0]}=-x_{1}{ }^{[0]}+u^{[0]} \quad, x_{2}{ }^{[0]}(0)=0
\end{gathered}
$$

and for $i \geq 1$

Minimize

$$
J^{[i]}=\frac{1}{2} \int_{0}^{5}\left(\left(x_{1}^{[i]}\right)^{2}+\left(x_{2}^{[i]}\right)^{2}+\left(u^{[i]}\right)^{2}\right) d t
$$

subject to

$$
\begin{aligned}
& \dot{x}_{1}{ }^{[i]}=x_{2}{ }^{[i]} \quad, x_{1}{ }^{[i]}(0)=1 \\
& \dot{x}_{2}{ }^{[i]}=-x_{1}{ }^{[i]}+\left(1-\left(x_{1}^{[i-1]}\right)^{2}\right) x_{2}{ }^{[i]}+u^{[i]} \\
& x_{2}{ }^{[i]}(0)=0
\end{aligned}
$$

After changing the time interval $t \in[0,5]$ to the interval $\tau \in[-1,1], x_{1}(\tau)$ is approximated by a $9^{\text {th }}$ order Chebyshev series, $x_{2}(\tau)$ is determined from (42) while $u(\tau)$ is determined from (40). The obtained values of $x_{1}(\tau), x_{2}(\tau)$ and $u(\tau)$ of the first iteration are substituted into (42)-(43) to solve for the next iteration, after approximating $x_{1}(\tau)$ by 9 th order Chebyshev series, $x_{2}(\tau)$ and $u(\tau)$ are obtained from (42) and (43) respectively.

Table 1 illustrates the results of the optimal values of the cost function $I$ verses iteration $i$ for Chebyshev polynomial with $N=9$ and $N=15$. Table 2 shows the optimal values $/$ obtained using some other methods for comparison purposes.

Fig. 1 shows the optimal trajectories of the Van der Pol oscillator problem.

Table 1. approximated optimal valud

\begin{tabular}{|c|c|c|}
\hline iteration $\boldsymbol{i}$ & $\mathbf{N = 9}$ & $\mathbf{N}=\mathbf{1 5}$ \\
\hline 0 & 0.9533622676 & 0.9533622624 \\
\hline 1 & 1.4516540238 & 1.4515286014 \\
\hline 2 & 1.4497313124 & 1.4496981112 \\
\hline 3 & 1.4493918353 & 1.4493287644 \\
\hline 4 & 1.4494606241 & 1.4494047276 \\
\hline 5 & 1.4494528889 & 1.4493959719 \\
\hline
\end{tabular}

Table 2. values of $J$ obtained using other methods

\begin{tabular}{|l|c|c|}
\hline \multicolumn{1}{|c|}{ Source } & $\mathrm{J}$ & Method \\
\hline Jaddu [3] & 1.433487 & $\begin{array}{c}\text { Quasilinearization and } \\
\text { state parameterization }\end{array}$ \\
\hline $\begin{array}{l}\text { Bullock and } \\
\text { Franklin [17] }\end{array}$ & 1.433508 & Second variation \\
\hline $\begin{array}{l}\text { Bashein and } \\
\text { Enns [18] }\end{array}$ & 1.438097 & $\begin{array}{l}\text { Quasilinearization } \\
\text { and discretization }\end{array}$ \\
\hline
\end{tabular}

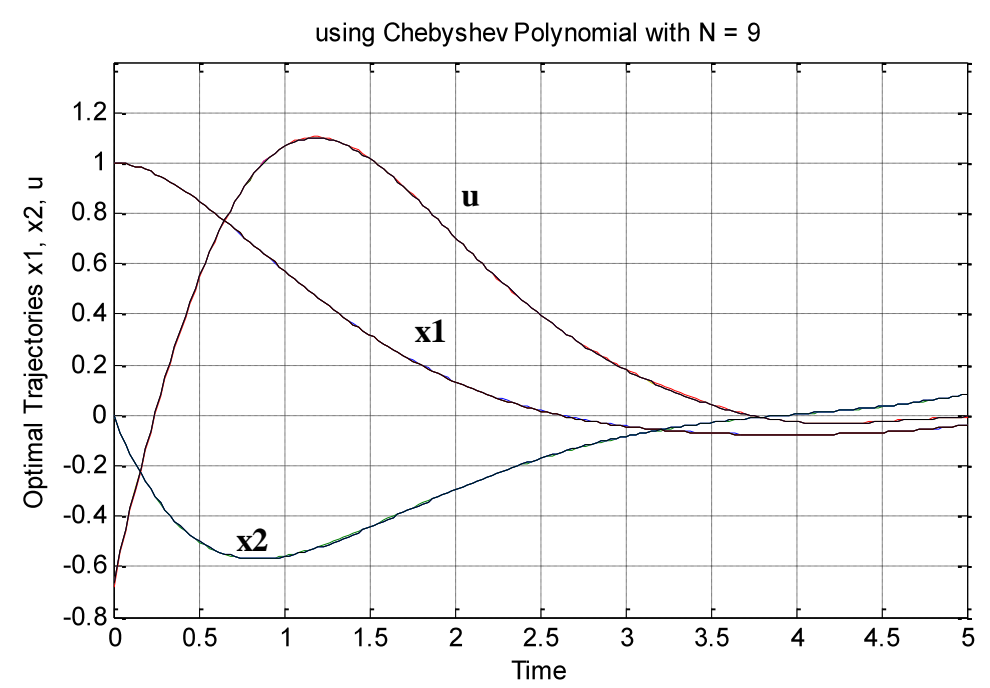

Fig. 1. Optimal trajectories of Van der Pol problem using Chebyshev polynomials 


\section{CONCLUSION}

In this work, a method for solving a class of nonlinear quadratic optimal control problem has been proposed. This method is based on replacing the nonlinear state equation by a sequence of linear time-varying state equations and then parameterizing the system state variables by a finite length Chebyshev series with unknown parameters.

To show the effectiveness of the proposed method, a Van der Pol oscillator problem is solved and the simulation results obtained indicate that the proposed method gives good and comparable results with some other methods.

\section{REFERENCES}

[1] Kraft D. On converting optimal control problems into nonlinear programming problems. Computational Mathematical Programming. Vol 15, Ed. Schittkowski, Springer, Berlin, 1985, pp. 261-280.

[2] Betts J. Issues in the direct transcription of optimal control problem to sparse nonlinear programs. Computational Optimal Control, Ed. R. Bulirsch and D. Kraft, Birkhauser, Germany, 1994, pp.3-17.

[3] Jaddu, H., Numerical methods for solving optimal control problems using Chebyshev polynomials, PHD Thesis 1998.

[4] Jaddu, H., Direct solution of nonlinear optimal control problems using quasilinearization and Chebyshev polynomials, Journal of the Franklin Institute, Vol. 339, 2002, pp 479-498.

[5] Jaddu, H and Vlach M. Successive approximation method for non-linear optimal control problems with applications to a container crane problem, Optimal Control Applications and Methods, Vol. 23, 2002, pp 275-288.

[6] Spangelo I. Trajectory optimization for vehicles using control parameterization and nonlinear programming. Phd thesis, 1994.

[7] Goh C J. and Teo K L. Control parameterization: A unified approach to optimal control problems with general constraints, Automatica, 24-1, 1988, pp 3-18.

[8] Vlassenbroeck J and Van Doreen R. A Chebyshev technique for solving nonlinear optimal control problems, IEEE Trans. Automat. Cont., 33,1988, pp 333-340.

[9] Frick P A and Stech D J. Epsilon-Ritz method for solving optimal control problems: Useful parallel solution method, Journal of Optimization Theory and Applications, Vol. 79, 1993, pp 31-58.

[10] Tomas-Rodriguez $\mathrm{M}$ and Banks S P. Linear approximations to nonlinear dynamical systems with applications to stability and spectral theory, IMA Journal of Control and Information, 20, 2003, pp 1-15.

[11] Banks S P and Dinesh, K. Approximate optimal control and stability of nonlinear finite and infinite-dimensional systems. Ann. Op. Res., 98, 2000, pp 19-44.

[12] Tomas-Rodriguez M and Banks S P. An iterative approach to eigenvalue assignment for nonlinear systems, Proceedings of the 45th IEEE Conference on Decision \& Control, 2006, pp 977-982.

[13] Navarro Hernandez C, Banks S P and Aldeen M. Observer design for nonlinear systems using linear approximations. IMA J. Math. Cont Inf(20), 2003, pp359-370
[14] Tomas-Rodriguez M, Banks S P and M.U. Salamci M U. Sliding mode control for nonlinear systems: An iterative approach, Proceedings of the 45th IEEE Conference on Decision \& Control, 2006, pp 4963-4968

[15] Jaddu $\mathrm{H}$ and Shimemura E. Computation of optimal control trajectories using Chebyshev polynomials: parameterization and quadratic programming, Optimal Control Applications and methods, 20, 1999, pp.21-42

[16] Fox L and Parker I B. Chebyshev Polynomials in Numerical Analysis, Oxford University Press, England, 1968.

[17] Bullock T and Franklin G. A second order feedback method for optimal control computations, IEEE Trans. Automat. Cont., Vol. 12, 1967, pp 666-673.

[18] Bashein G and Enns M. Computation of optimal control by a method combining quasi-linearization and quadratic programming, Int. J. Control, Vol. 16, 1972, pp 177-187.

\section{Authors' Profiles}

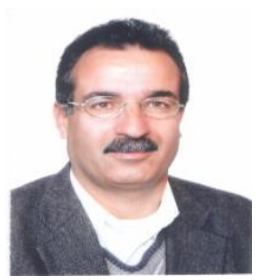

Hussein Jaddu received his BSc. in Electrical Engineering from BirZeit University, Palestine, in 1987, MSc. in Electronics (dig ital systems) from Hatfield Polytechnic in England 1991, Phd in information Systems from Japan Advanced Institute of Science and Technology JAIST, Japan 1998. He worked as a research associate at JAIST during the period 20002002. He is with Electronics Engineering Department of Al Quds University in Palestine where he works as an associate professor. In addition to the academic position he also serve as the university vice president for administrative and financial affairs. His main areas of research interests are computational of optimal control, wavelets, congestion control, obstacle avoidance.

Amjad Majdalawi received his BSc. in Electronics Engineering from Yarmouk University, Jordan, in 1997. He then worked in several private sector factories as an electrical/control engineer. In 2010, he received his MSc. degree in electronics and computer engineering from $\mathrm{Al}$ Quds University. He is currently working at the ministry of education and higher education in Palestine in the technical education, His main areas of research interests are computational of optimal control, digital communication, and SCADA systems.

How to cite this paper: Hussein Jaddu, Amjad Majdalawi,"An Iterative Technique for Solving a Class of Nonlinear Quadratic Optimal Control Problems Using Chebyshev Polynomials", IJISA, vol.6, no.6, pp.53-57, 2014. DOI: 10.5815/ijisa.2014.06.06 